\title{
THE PROPAGATION OF UNCERTAINTY THROUGH TRAVEL DEMAND MODELS: AN EXPLORATORY ANALYSIS
}

\author{
Yong Zhao, PhD Candidate \\ Dept. of Civil Engineering - The University of Texas at Austin \\ E-mail: yzhao@mail.utexas.edu \\ Kara Maria Kockelman (Corresponding Author) \\ Clare Boothe Luce Professor of Civil Engineering, The University of Texas at Austin \\ Email: kkockelm@mail.utexas.edu
}

\section{INTRODUCTION}

The future operations of the transportation system involve enormous uncertainty. Modeling these complicated systems requires many variables and behavioral components whose variability may be poorly identified or simply ignored. Generally, large-scale transport demand models are estimated sequentially, with the results or estimates of one model acting as inputs to subsequent models. In almost all cases, only point estimates are passed forward, rather than estimates of variation and covariation. Such modeling processes limit the final results to point estimates, so comparisons of plans or scenarios based on the results may be incorrect. This work investigates the nature of uncertainty propagation in contemporary transport demand models, by quantifying variability in model outputs and tracking the sources of this variability from model inputs' and parameters' variations. Monte Carlo simulation and sensitivity analysis are used to investigate error propagation over an 818-link network covering a 25-zone area of the Dallas-Fort Worth metro region.

Current travel-demand-modeling practice does not acknowledge the sources of stochastic uncertainty, especially input uncertainty. For example, rigorous statistical models produce estimates of variance and covariance with their point (or mean) estimates. However, only point estimates (of variables' mean values) are carried forward through travel demand models. The covariance information is generally lost. Many variables used as input in transport demand models come from other models, whose associated uncertainty is not known or incorporated. If point estimates of these future variables (such as population, housing, and automobile ownership) are to be used in travel demand models, an appreciation of variability in all results requires distributional information on the input.

Modeling methods based on point estimates dramatically constrain all final results into point estimates, and the point estimates may be highly biased. Comparisons of alternative 
transportation plans or scenarios based on these do not convey information regarding uncertainty in estimates - or the statistical significance of differences. Neglect of data and parameter uncertainties and their correlation ultimately weaken the reliability of transportation planning, policy-making, and infrastructure decisions. For example, Rodier and Johnston (2001) suggested that the plausible uncertainty in population and employment projections may result in a region's transportation plan not meeting the air quality conformity tests in a five or ten year time horizon.

To assess some forms of uncertainty in model predictions, most transport modeling processes employ "model validation" to test a model's forecast ability. Variations in future forecasts due to input and inherent uncertainty, however, change over time. Thus, there is no guarantee that future predictions will be bounded by an acceptable range. A "before and after" study is another method used to assess a model's predictive accuracy. It also is difficult to draw useful conclusions from an individual study (Aitken and White, 1972).

There is a fair amount of transportation research really focused on modeling uncertainties. Most of them adopted a simulation technique to capture uncertainty patterns. Using simulation techniques, Ashley (1980) studied the probability distribution of various outputs from an interurban highway forecasting model due to various input uncertainties. His correlated inputs were drawn from multivariate probability distributions; but he neglected many forms of uncertainty (e.g., destination choice), did not detail the specifics of his simulations, and did not investigate which error sources contributed most to overall uncertainty. In contrast, Pell (1984) suggested a method to estimate variability in travel demand forecasts based on identifying those sources of input uncertainty and error that make the largest contributions to forecast uncertainty. His 73 simulations suggested link-flow coefficients of variation of 0.30 to 2.0 , but he did not employ correlated inputs.

Simulation techniques are suggested as one of the most useful methods in this field because one can simulate uncertainty from a variety of sources simultaneously. Sensitivity analysis is another effective tool for studying uncertainty. It traces output uncertainty back to inputs, revealing both linear and non-linear relationships. However, due to cost, computational, and other limitations, prior studies exhibit common weaknesses. Few large-scale data applications have been undertaken, and few firm conclusions have been reached.

\section{METHODOLOGY}

This study adopts the effective methods suggested by prior researches. It investigates the stability of transportation demand model outputs by using traditional, four-step urban transportation planning process (UTPP) models. These variations studied in this work are 
purely stochastic; they may or may not be related to actual human behaviors' diversities. (And such behavioral comparison would be addressed in later studies.) Monte Carlo simulation and sensitivity analysis are the primary tools used here. A multivariate regression analysis of results (as a function of input levels) along with linear and rank correlation coefficients suggests dependencies and sensitivities between input and output uncertainties

This work considers the traditional UTPP model paradigm via its primary components: trip generation, trip distribution, mode choice, and route selection. There are a number of alternative model formulations and specifications for each stage. However, as a demonstration to reveal the uncertainty propagation, this study only adopts simplified model specifications. It uses a crossclassification model to calculate the home based work trips (HBW) in trip generation. Three types of employment are used: basic employment, retail employment, and service employment. Four types of zones are specified based on the population and employment density. They are business district, urban residential, suburban residential, and rural. A common gravity model form is used for trip distribution, with a production constraint. A simple exponential function is used as the impedance function for the gravity model. This study simplifies the travel mode choice between drive-alone and all other modes (based on public transit travel times) and uses a binary logit model for mode split. It employs a user equilibrium method in its trip assignment model and incorporates a standard Bureau of Public Roads link capacity function in searching for convergence to an equilibrium state. This study applies the larger BPR parameter values as NCHRP report 365 suggested.

All together, this sequence of four sub-models produces a set of link-flow estimates. These are the model outputs of greatest interest in this work, and their variability is due solely to input and parameter uncertainties. The four-step model approach is applied into a road network with 25 zones and 818 links, which is separated from the Dallas-Fort Worth highway system. For outside input, this study uses the demographic data associated with the data. For model parameters, this study uses mean values from DFW area travel model description report (NCTCOG 1999). Necessary simplification and modification has been made based on two published formal manuals (NCHRP Report 187 and 365). However, there are several arbitrary variation and covariation assumptions; these include a single coefficient of variation for all inputs and parameters and a single correlation coefficient (of +0.30 ) relating all demographic data inputs.

The modeling software used here for the first three sub-model steps (i.e., trip generation, trip distribution, and mode choice) is @Risk (Palisade 1998), which loads through Microsoft Excel software, for Monte Carlo simulation and risk analysis. TransCAD is used here for the final, trip assignment sub-model in order to apply its commercialized UE algorithm. 


\section{EMPIRICAL RESULTS}

The sequence of four-step sub-models produces a set of link-flow estimates. The study simulates the forecasting approach by repeating running the four-step models for 100 times. Two example arcs are chosen for explicit consideration. Link one presents the general pattern of links with congested volumes, while link two presents others, uncongested links. All the uncertainty in terms of coefficient of variation (COV) were set to 0.30. As evident in these results, the variability of the selected link flows is sizable. Both coefficients of variation of the two link flows are larger than 0.30 , which suggests the final uncertainty may be compounded to end higher than any input or parameter uncertainty. The flow uncertainty appears not to have a strong relation with congestion; most link flow uncertainties are larger than 0.30, no matter what their v/c ratios are. However, the average travel time on the link shows a relatively strong relation with congestion. The travel time uncertainty of the example congested link, 1.899, is much higher than that of the uncongested link, 0.127. The COV estimated of VMT is just 0.236 , which is relatively low. The link flows show great correlation between one another. For probabilistic simulations, correlations greater than 0.5 between input and output indicate substantial dependence. Since the total VMT is the weighed sum of all link flow volumes, there is a strong correlation between total VMT and individual link flows.

In each model step, there is a finite amount of input and output. Given the distribution assumption of the input and parameters of the model, the simulation yields 100 observations of each output. Although the amount of output of each step is different, the average COV, as a scaleless measurement, can be collected to track the changes in uncertainty through model stages. The five percentile and ninety-five percentile of the uncertainty among each step are also collected to indicate the variability of the uncertainty, as shown in Fig. 1. As can be seen, the increasing average uncertainty in the first three step models suggests significant uncertainty propagation through those models. Nevertheless, the final step assignment model somehow reduces the previous compounded uncertainty, but generally not lower than the input uncertainty. The expanding 5\% and $95 \%$ bound suggests that through the four-step model, the variability of final uncertainty extends. Thus, some link flows' uncertainty may be reduced substantially while others may increase considerably, which indicates the possibility for wide swings in system. However, one still can improve the UTPP model forecasting by providing the information regarding uncertainty in final results. In this way, the policymakers will be aware of the uncertainty when comparing scenarios. The simulation results suggest the trip assignment equilibrium technique may reduce the overall uncertainty, which is partially consistent with Leurent's (1998) study. 


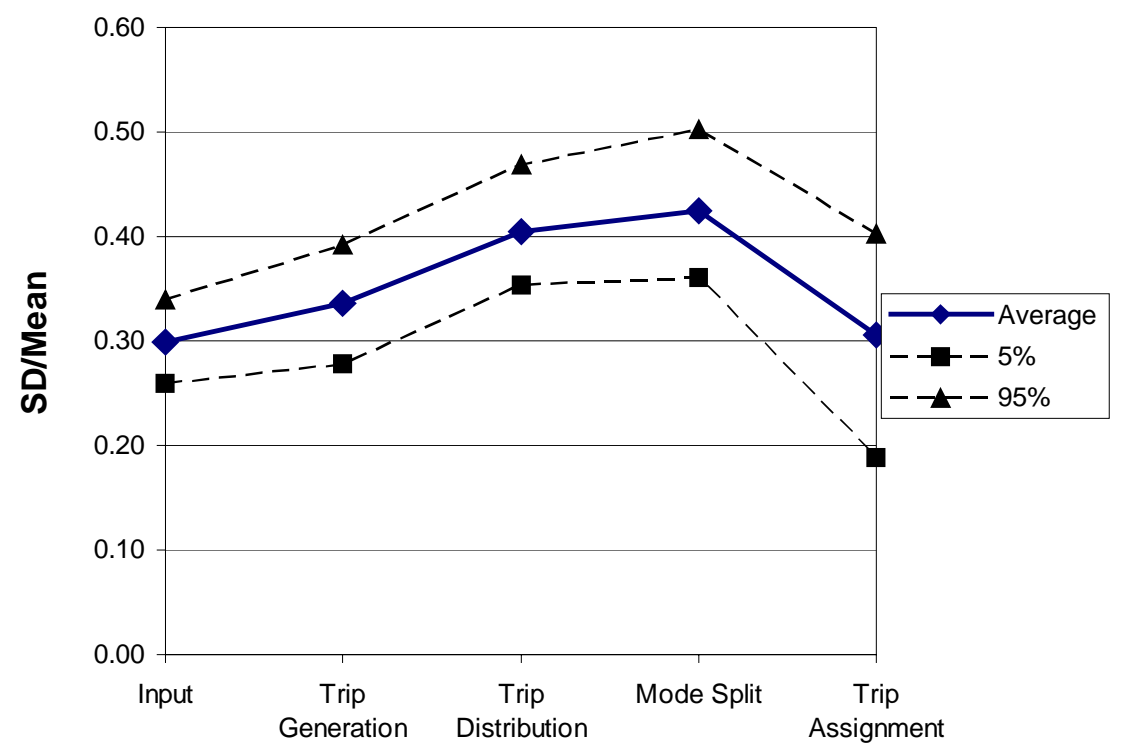

Fig. 1. Uncertainty propagation through 4-step models

For better understanding and interpretation of the four-step model results, sensitivity analysis was used to identify model inputs that are key contributors to uncertainty in model output (see Fig. 2). Not surprisingly, the parameter which has the strongest correlation with link flows is the trip generation rate. This is partly consistent with Smith and Cleveland's results (1976). Also, the overall output is sensitive to the demographic input. Most zonal demographic inputs contribute substantially to the overall uncertainty in link flows. Given the linear function pattern of the trip generation model, it is not surprised that the demographic inputs and the trip generation parameters show strong linear correlation with the overall outputs. Moreover, the rank correlation coefficients show the non-linear correlation between inputs and outputs. The results are somewhat similar to the linear correlation analysis. The trip attraction rates of basic and service employments for land use type 3 (suburban residential) show stronger correlation to final results than other parameters in trip generation, because most zones in this study area belongs to suburban residential and basic and service employments are the main employment types in these zones. In addition, the parameters in mode split are found to play important roles in result variation. In contrast, results variations exhibit little sensitivity to the parameters of the trip distribution and trip assignment models; this result may be due to the less-thanstraightforward application of those models due to iterative trip-balancing for trip distribution and user-equilibrium feedbacks used in trip assignment. 


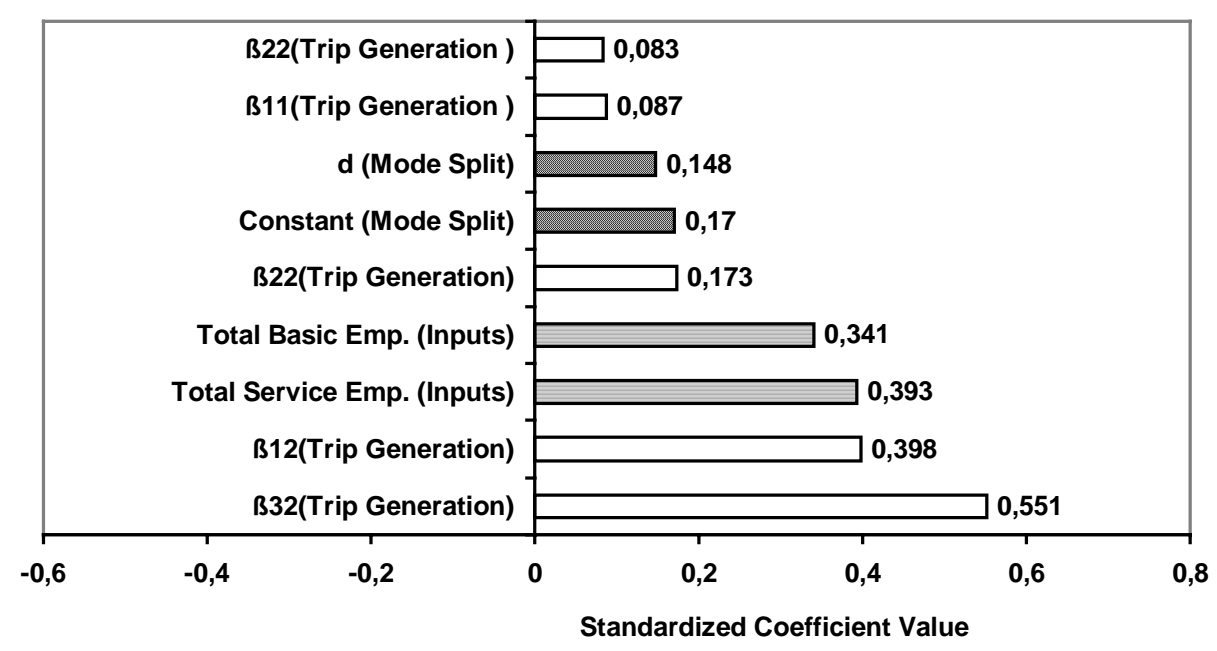

Fig. 2. Regression-based sensitivity analysis for final outputs

\section{FUTURE RESEARCH DIRECTIONS}

Future work on this and related topic is needed. For example, applications on more realistic networks may be examined. A variety of common model types can be tested. In addition, feedbacks of travel-time estimates to destination, mode, and route choices would be valuable. Also, factorized "experiments" rather than random simulations may be more efficient at sampling the set of possible environments and distinguishing the contributions and interactions of different random inputs and parameters. Such work will help identify which aspects of modeling practice are the biggest contributors to result uncertainty - and where modeling improvements are likely to be most effective for added precision.

In general, since inputs and parameter estimates are uncertain, transportation modelers would do better to recognize, estimate, and specify result uncertainties. In addition, policymakers should appreciate these uncertainties and incorporate such information in their decisionmaking. This work represents a step in this direction. 


\section{REFERENCES}

Aitken, J.M. and R. White (1972). A Comparison between a Traffic Forecast and Reality. Traffic Engineering and Control. August, pp.174-177.

Ashley, D.J. (1980). Uncertainty in the Contest of Highway Appraisal. Transportation 9(3), pp. 249267.

Leurent, F. (1998). Sensitivity and Error Analysis of the Dual Criteria Traffic Assignment Model. Transportation Research, 32B(3), pp. 189-204.

Martin, W. (1998). Travel Estimation Techniques for Urban Planning. NCHRP Report 365, Transportation Research Board, National Research Council, Washington, D.C.

NCTCOG (1999) Dallas-Fort Worth Regional Travel Model (DFWRTM): Description Of The Multimodal Forecasting Process. Transportation Department, North Central Texas Council of Governments, Texas.

Palisade (1998). @Risk: Simulation Add-In For Microsoft Excel. Windows Version Release 4.0. Newfield, NY: Palisade Corp. (November)

Pell, C. M. (1984).The Analysis of Uncertainty in Urban Transportation Planning Forecasts. Ph.D. Dissertation, Cornell University.

Rodier, C. J. and R. A. Johnston (2001) Uncertainty Socioeconomic Projections Used in Travel and Emissions Models: Could Plausible Errors Result In Air Quality Nonconformity? Presented at the 80th Annual Meeting of the Transportation Research Board, Washington, D.C., January.

Sosslau, A.B., et al. (1978). Quick Response Urban Travel Estimation Techniques and Transferable Parameters: User Guide. NCHRP Report 187, Transportation Research Board, National Research Council, Washington, D.C. 